УДК 62-69

\title{
ЭФФЕКТИВНЫЕ ХАРАКТЕРИСТИКИ ТЕПЛООБМЕНА В ПОРИСТОМ СЛОЕ ТЕПЛОГЕНЕРАТОРА БЕСПЛАМЕННОГО ГОРЕНИЯ
}

\author{
Хаустов Сергей Александрович', \\ khaustovSA@tpu.ru \\ Вислогузов Руслан Ахмедович', \\ visloguzov.ra@yandex.ru \\ Ивашутенко Александр Сергеевич', \\ ivashutenko@tpu.ru
}

Долгов Сергей Викторович², sergeydolgov555@rambler.ru

\author{
Табакаев Роман Борисович', \\ TabakaevRB@tpu.ru
}

\author{
Ермолаев Антон Николаевич, \\ ermolaevanton03@gmail.com \\ 1 Национальный исследовательский Томский политехнический университет,
Россия, 634050, г. Томск, пр. Ленина, 30. \\ 2 Муниципальное унитарное предприятие г. Нижневартовска «Теплоснабжение», \\ Россия, 628616, г. Нижневартовск, ул. Менделеева, 21. \\ 3 Тюменский индустриальный университет, \\ Россия, 625000, г. Тюмень, ул. Володарского, 38.
}

Одним из наиболее распространенных и опасных выбросов, загрязняющих биосферу, являются жидкие углеводородные отходы. В их составе может содержаться более 200 опасных соединений, а колоссальные темпы образования производственных отходов привели к накоплению человечеством более 1 млрд т различных жидких техногенных отходов. В связи с этим назрела проблема утилизации таких жидких отходов, решение которой является одной из приоритетных задач, стоящих перед мировым сообществом. Перспективным направлением в решении этой проблемы является огневая утилизация жидких отходов в беспламенном режиме. Беспламенный режим горения обеспечивается сжиганием жидкого топлива в пористой матрице инертного наполнителя, в качестве которого использован криптол. Актуальной задачей для развития этого научного направлении и конструкторской разработки связанных с ним технических решений является исследование эффективных характеристик криптоловой матрицы.

Цель работы: определение теплофизических характеристик углеродной матрицы криптола и получение аналитических зависимостей для инженерных расчетов её предварительного прогрева.

Методы исследования: измерение истинных значений теплоемкости и теплопроводности криптола на анализаторе температуропроводности Discovery Flash DLF-1200 в диапазоне температур 298-573 K. Теплофизические процессы при прогреве криптоловой пористой матрицы плоским нагревательным элементом экспериментально исследовались на специально разработанном экспериментальном стенде. Рассмотрено два варианта компоновки стенда: с минимальной и максимальной интенсивностью свободной конвекции воздуха в порах. При математической обработке экспериментальных данных эффективное значение коэффициента температуропроводности выражалось из конечноразностного аналога дифференциального уравнения теплового баланса в нестационарной постановке.

Результаты: получены аналитические зависимости эффективного значения температуропроводности от температуры, позволяющие рассчитывать процесс предварительного прогрева криптоловой матрицы с применением дифференциального уравнения теплопроводности, для определения расположения и необходимой мощности нагревательных элементов.

\section{Ключевые слова:}

Жидкие углеводородные отходы, огневая утилизация, пористый слой, криптол,

теплогенератор, эффективная температуропроводность.

\section{Введение}

Изменение климата, вызванное загрязнением окружающей среды и выбросом парниковых газов, является глобальной угрозой для нашей планеты, оказывая огромное влияние на здоровье человека и состояние окружающей среды [1-4]. Исследования Международного энергетического агентства
(International Energy Agency, IEA) показали, что в результате загрязнения воздуха в мире каждый день умирает около 18000 человек [1]. Главными источниками загрязнения окружающей среды попрежнему остаются энергетика и промышленность $[1,5]$. При этом негативному влиянию вредных выбросов подвержена не только биосфера, но и тех- 
нические объекты: кислотные дожди приводят к коррозии металлических сооружений, выбросы золы являются причиной повышенного износа конструкций, проникновение химически агрессивных жидких отходов в почву влечет за собой ускоренную коррозию коммуникаций [6].

Жидкие углеводородные отходы, образующиеся в технологических процессах выработки электроэнергии и производства, являются одним из наиболее распространенных и опасных выбросов. В их составе может содержаться более 200 опасных соединений [7], способных нанести непоправимый вред человеку и окружающей среде. Основными источниками подобных отходов выступают нефтеперерабатывающие предприятия, химическая промышленность и автомобильный транспорт. По оценке экспертов [8-12], ежегодно в мире образуется около 30-40 млн т автомобильного и 32-35 млн т индустриального отработанных масел, 60 млн т нефтешламов и др. Столь колоссальные темпы образования отходов привели к тому, что по данным [13] на 2013 г. уже накоплено более 1 млрд т различных жидких техногенных отходов. В связи с этим назрела проблема утилизации жидких углеводородных отходов, решение которой является одной из приоритетных задач, решаемых мировым сообществом.

Среди возможных путей утилизации жидких углеводородных отходов можно выделить огневое сжигание, которое одновременно позволяет решить две задачи - непосредственную утилизацию жидких отходов и энергообеспечение потребителей. При этом основными проблемами этого напра-

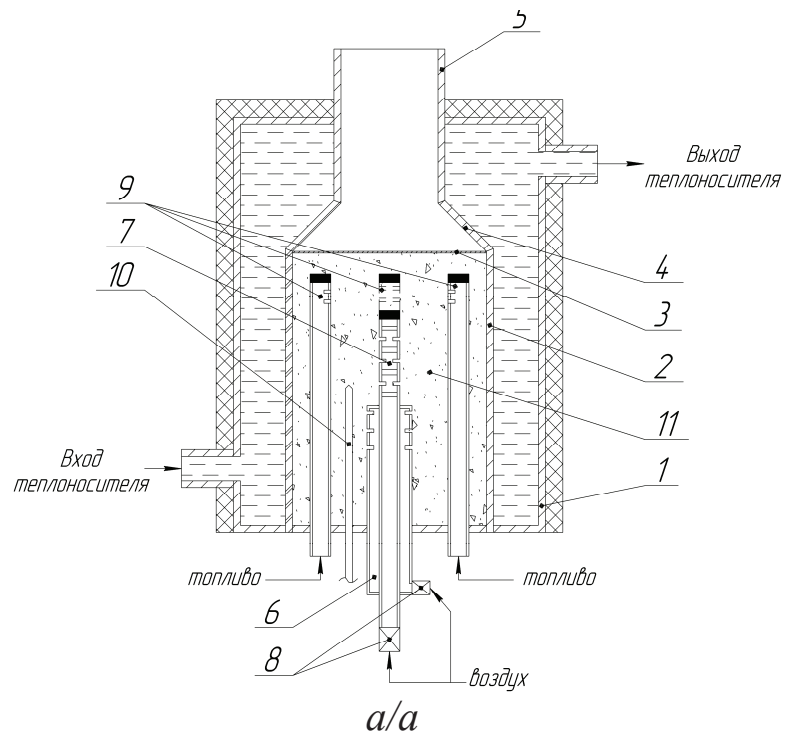

вления являются: обеспечение экологичности, стабильности горения и взрывопожаробезопасности $[14,15]$.

В качестве одного из перспективных решений перечисленных проблем известна огневая утилизация жидких углеводородных отходов в беспламенном режиме. Беспламенный режим горения, как правило, осуществляется путем каталитического окисления или созданием условий стехиометрического горения топливовоздушной смеси в пористой структуре [16-19]. В [20] отмечается, что в беспламенных горелках процесс горения имеет специфический характер: продукты сгорания уже в зоне горения отдают значительное количество теплоты и охлаждаются, что не позволяет протекать реакциям окисления азота по термическому механизму. Доля $\mathrm{CO}_{\mathrm{x}}$ и $\mathrm{NO}_{\mathrm{x}}$ в уходящих газах при работе беспламенных горелок значительно ниже, чем при факельном сжигании [21-24].

Примерами промышленного внедрения беспламенных горелок являются газовые горелки Riello (Италия) и MatriX-Gasbrenner (Viessmann, Германия) $[20,25]$. Однако для сжигания жидких углеводородных отходов перечисленные горелочные устройства не предназначены. В качестве технического решения, позволяющего сжигать жидкие углеводородные отходы в беспламенном режиме, может быть рассмотрен инфракрасный теплогенератор (рис. $1, a$ ), описанный в работе [26]. Принцип работы устройства заключается в сжигании жидкого топлива в пористой матрице инертного наполнителя, в качестве которого использован отход сталелитейного производства - криптол.

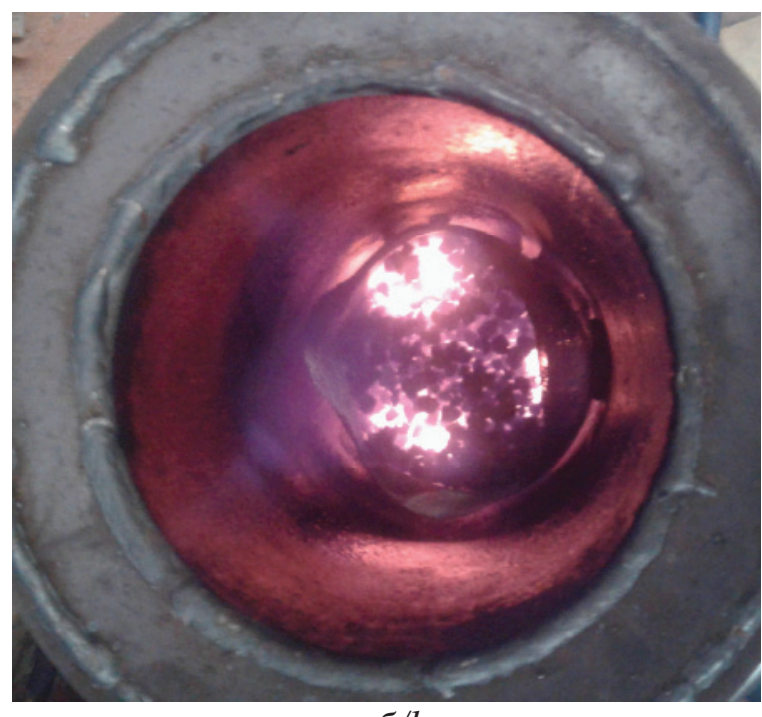

$\sigma / b$

Pис. 1. Устройство для получения тепла за счет сжигания жидких отходов углеводородного состава в беспламенном режиме (а) и вид его камеры сгорания в процессе испытаний (б): 1 - корпус; 2 - камера сгорания; 3 - металлическая сетка; 4 конвективная часть; 5 - дымовая труба; 6 - первый воздушный инжектор; 7 - второй воздушный инжектор; 8 - регулировочная арматура; 9 - топливные инжектора; 10 - запальное устройство; 13 - засыпка криптола

Fig. 1. Device for obtaining heat by burning liquid hydrocarbon wastes in a flameless mode (a) and its combustion chamber during the test (b): 1 is the casing; 2 is the combustion chamber; 3 is the metal mesh; 4 is the convective part; 5 is the chimney; 6 is the the first air injector; 7 is the second air injector; 8 is the adjusting valve; 9 is the fuel injectors; 10 is the ignition device; 13 is the cryptol filler 
Предварительные результаты численных и экспериментальных исследований [27, 28] показали работоспособность предложенного устройства и возможность осуществления его работы в беспламенном режиме (рис. 1, б). Однако существующая тепловая инерция криптола существенно усложняет организацию процесса воспламенения и стабильного горения углеводородных отходов. Дальнейшее развитие исследований в этом направлении и конструкторская разработка технических решений на его основе требуют знания как истинных теплофизических характеристик криптола, так и әффективных термических характеристик пористой засыпки криптола в условиях работы теплогенератора.

В частности, актуальной задачей является разработка способов инициирования воспламенения и совершенствование технологий предварительного прогрева криптоловой матрицы. В процессе предварительного прогрева ставится задача определения такого минимального количество тепла, которое необходимо сообщить криптоловой матрице, чтобы его было достаточно как для воспламенения топлива, так и дальнейшего поддержания горения. Кроме того, для прогнозирования неравновесных тепловых процессов в углеродной матрице криптола требуется определить набор её теплофизических величин, характеризующих скорость изменения температуры среды (температуропроводность, теплоемкость). В литературе [29] изменение значений этих параметров однозначно связывается с изменениями самой температуры.
В связи с вышеперечисленным целью настоящей работы ставится определение теплофизических характеристик углеродной матрицы криптола и получение аналитических зависимостей для инженерных расчетов её предварительного прогрева.

\section{Объект и метод исследования}

Согласно рекомендациям [30], в качестве объекта исследования рассмотрена криптоловая матрица фракцией 5-10 мм. Для проведения экспериментов по определению теплофизических характеристик криптола предварительно определены истинная и насыпная плотности.

Истинная плотность ( $\left.\rho_{\text {исг }}\right)$ измерена отношением массы криптолового образца, изготовленного прессованием $(P=314 \mathrm{MПа)} \mathrm{измельченного} \mathrm{крип-}$ тола с добавлением $5 \%$-го раствора декстрина в воде, к его объему после достижения воздушно-сухого состояния. Значение истинной плотности составило 1418,9 кг/ $\mathrm{m}^{3}$.

Исследование истинных значений теплоемкости и теплопроводности криптола проведено на анализаторе температуропроводности Discovery Laser Flash DLF-1200 (TA Instruments, США) в диапазоне температур 298-573 K. Верхний предел температур обусловлен величиной температуры вспышки отработанного масла ( 598 K [31]).

По порядку экспериментально определенной величины истинного коэффициента температуропроводности $a \approx 10^{-7} \mathrm{M}^{2} / \mathrm{c}$ (рис. 2) можно характеризовать криптол как теплоизолирующий материал. Однако значения эффективных термических ха-

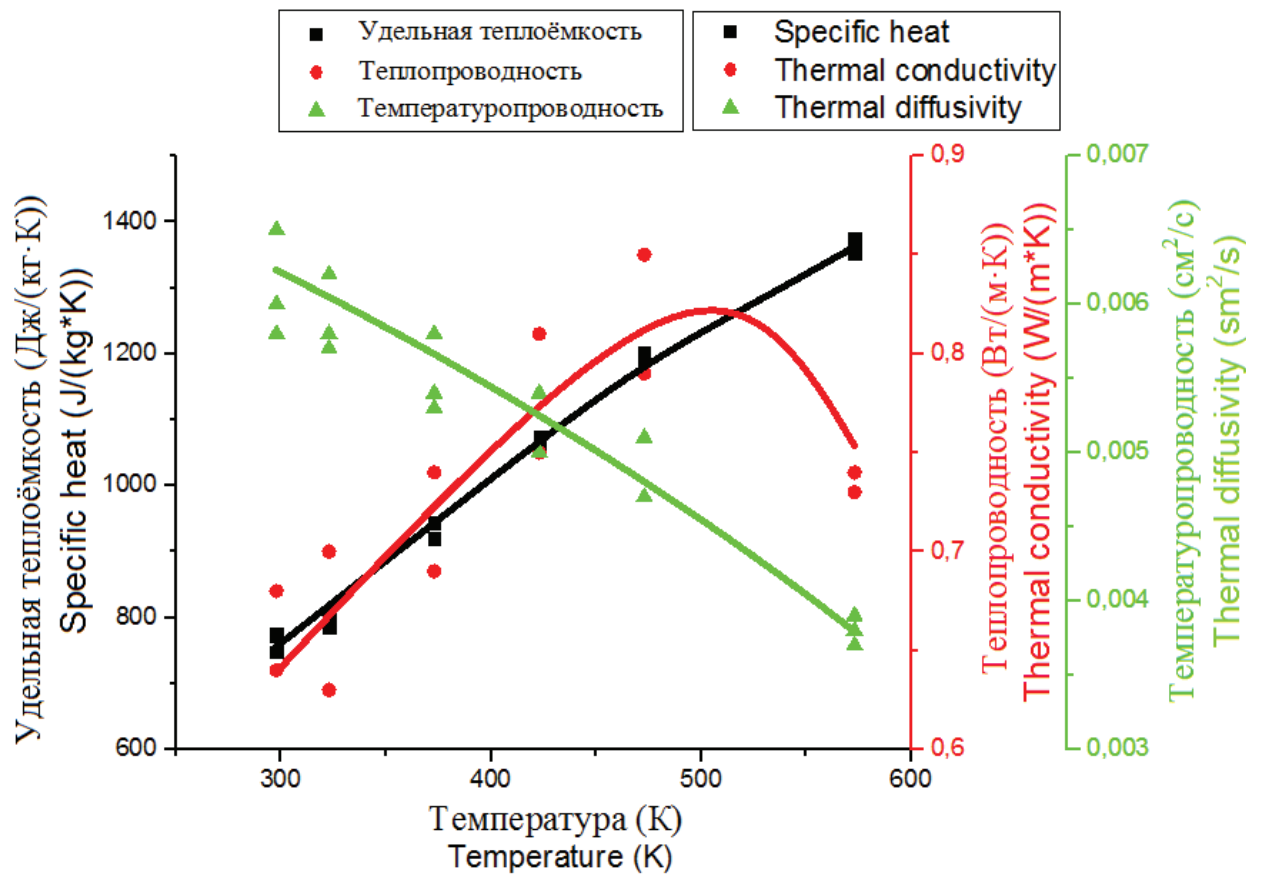

Рис. 2. Измеренные истинные значения теплоемкости (Дж/(кг·К)), теплопроводности $(B T /(M \cdot K))$ и температуропроводности (см²/с) криптола

Fig. 2. Measured values of cryptol heat capacity $(\mathrm{J} /(\mathrm{kg} \cdot \mathrm{K}))$, thermal conductivity $(W /(m \cdot K))$ and thermal diffusivity $\left(\mathrm{cm}^{2} / \mathrm{s}\right)$ 
рактеристик (эффективного коэффициента температуропроводности, эффективной теплоемкости) при изучении тепломассообмена в пористой среде зависят от её проницаемости, рабочих условий процесса нагрева и, как правило, отличаются от значений истинных теплофизических характеристик материала. В связи с этим при конструировании, компоновке и определении режимов работы теплогенераторов с пористым наполнителем представляют значительный интерес экспериментальные исследования их работы в стендовых условиях.

Теплофизические процессы при прогреве криптоловой пористой матрицы плоским нагревательным элементом исследовались на специально разработанном экспериментальном стенде (рис. 3).
Изолированная металлическая труба - 2 (диаметр 169 мм, толщина стенки 5,5 мм) устанавливалась вертикально, после чего в неё засыпался криптол 1 (фракция 5-10 мм). В засыпанный слой криптола устанавливались измерительные термопары, соединенные с многоканальным технологическим регистратором - 5. Термопары расположены у поверхности нагревательного элемента $\left(T_{0}\right)$ и на трех уровнях по высоте $\left(T_{1}, T_{2}, T_{3}\right)$ с фиксированным шагом. Для контроля точности получаемых данных предусмотрены дублирующие термопары $T_{0}^{\prime}-T_{3}^{\prime}$, расположенные на тех же уровнях, что и измерительные.

Исследовались два варианта расположения нагревательного элемента. Он располагался горизонтально либо снизу (рис. $3, a)$, либо сверху (рис. 3, б) -

$a / a$

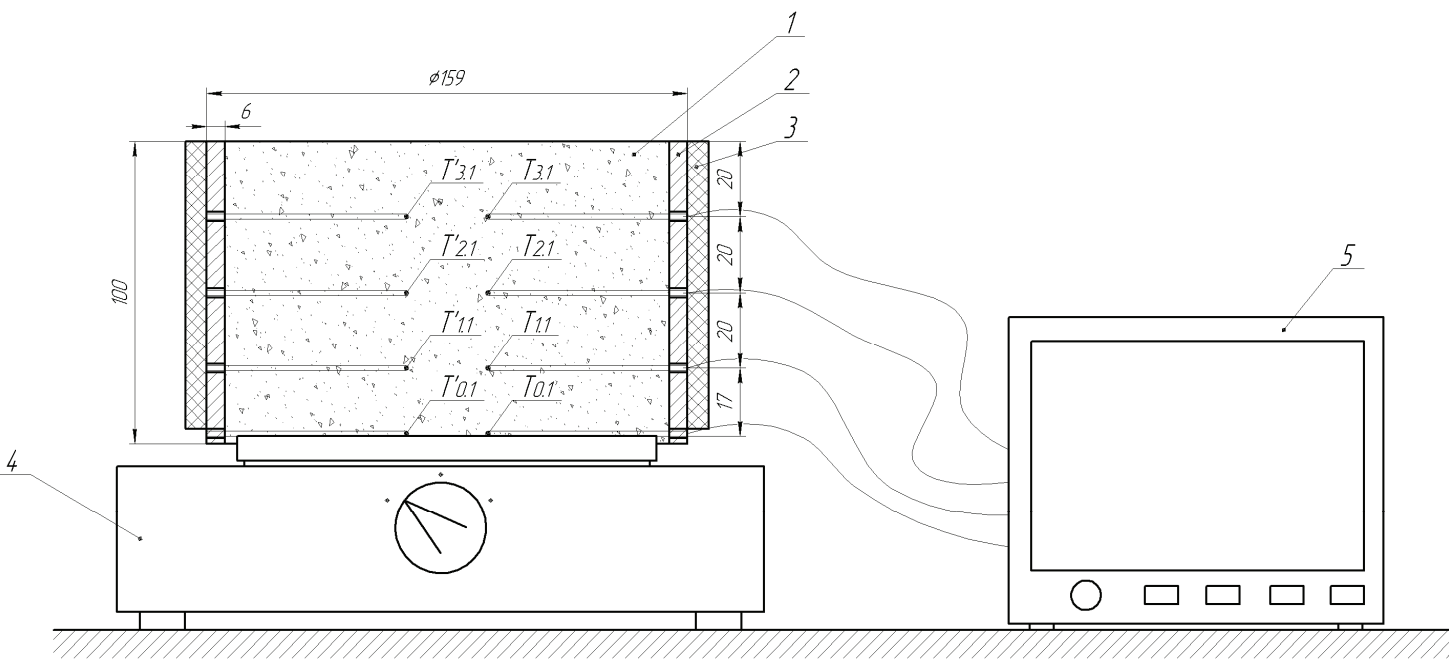

$\sigma / b$

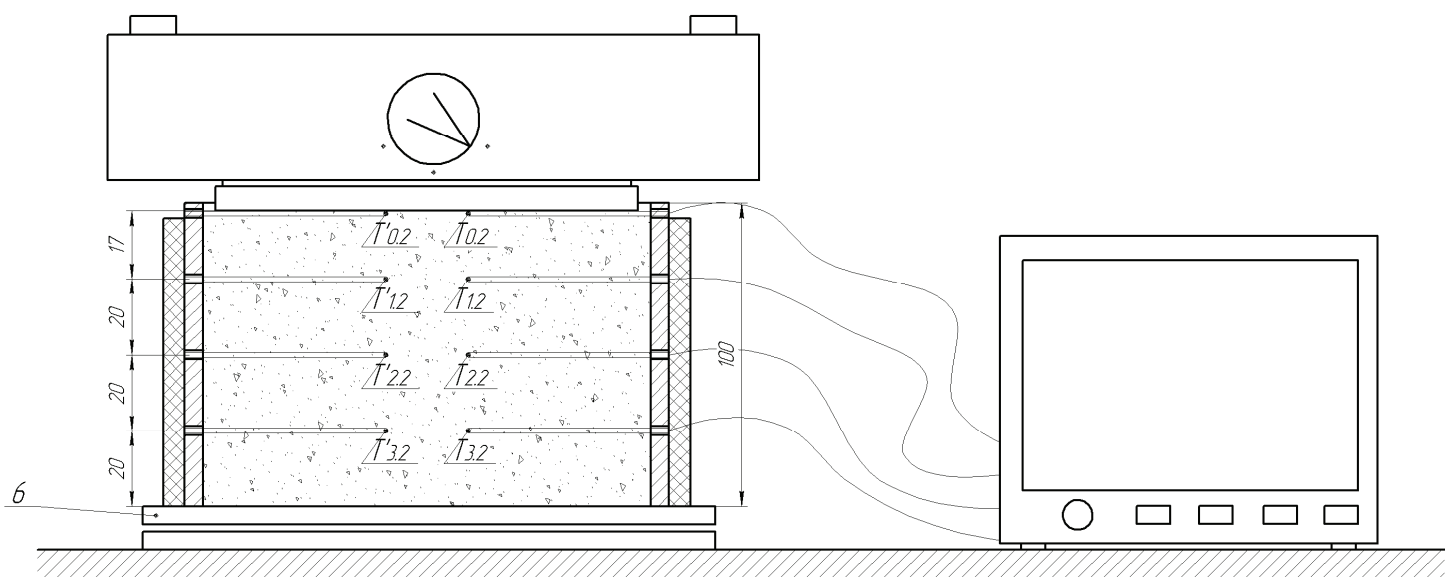

Рис. 3. Экспериментальный стенд для определения теплоемкости и теплопроводности криптола при различной ориентации подвода тепла: а) первый стенд (поверхность нагревательного элемента ориентирована вверх); б) второй стенд (поверхность нагревательного элемента ориентирована вниз); 1 - криптол; 2 - металлическая труба; 3 - теплоизоляция; 4 - нагревательный элемент; 5 - многоканальный технологический регистратор РМТ-59M (Элемер, Россия); 6 - крышка; $T_{0.1}-T_{3.1}-$ измерительные термопары (XА) первого стенда; $T_{0.2}-T_{3.2}-$ измерительные термопары (XА) второго стенда; $T_{0.1}^{\prime}-T_{3.1}^{\prime}, T_{0.2}^{\prime}-T_{3.2}^{\prime}$ - дублирующие термопары (XА) первого и второго стендов соответственно

Fig. 3. Experimental stand for determining cryptol heat capacity and thermal conductivity: a) the first stand (the heating surface is oriented upwards); b) the second stand (the heating surface is oriented downwards); 1 is the cryptol; 2 is the metal pipe; 3 is the thermal insulation; 4 is the heating surface; 5 is the multichannel technological recorder PMT-59M (Elemer, Russia); 6 is the cover; $T_{0.1}-T_{3.1}$ are the measuring thermocouples ( $K$-Type) of the first stand; $T_{0.2}-T_{3.2}$ are the measuring thermocouples ( $K$ - $T y$ pe) of the second stand; $T_{0.1}^{\prime}-T_{3.1}^{\prime}, T_{0.2}^{\prime}-T_{3.2}^{\prime}$ are the duplicating thermocouples (K-Type) of the first and second stand, respectively 
так, чтобы его поверхность была перпендикулярна оси металлической трубы. При такой компоновке вследствие неустойчивого распределения плотности воздуха под воздействием градиента температуры в порах возникают конвективные потоки. Критерий Нуссельта, характеризующий количественное соотношение между конвекцией и теплопроводностью в условиях неподвижной среды, зависит от ориентации поверхности нагревательного элемента -4 , и его расчетное значение для ориентированной вверх (рис. $3, a$ ) горячей поверхности в два раза выше, чем для ориентированной вниз (рис. 3, б) [29]. Таким образом, расположение и ориентация нагревательного элемента определяют условия конвективного теплообмена в порах, влияющие на его интенсивность.

Расчетные значения числа Нуссельта для обоих вариантов расположения нагревательного элемента не превышают 20, что характерно для ламинарных течений. При этом расчетное значение безразмерного критерия Рэлея, определяющего поведение воздуха при свободной конвекции, в обоих случаях имеет пятый или шестой порядок (на четыре порядка выше соответствующего значения температурного градиента).

При проведении эксперимента на стенде (рис. 3) непрерывно регистрировалась температура в трех точках на определенном расстоянии друг от друга и от плоского источника теплоты. Чтобы исключить влияние необратимости температурных изменений на изучаемые характеристики, эксперимент воспроизводился трижды как с полной заменой, так и без замены криптоловой засыпки между опытами. $\mathrm{K}$ моменту начала каждого эксперимента температура в объеме пористой засыпки распределялась равномерно и равнялась с температурой окружающей среды.

\section{Результаты исследования и их обсуждение}

Результаты проведенных испытаний на экспериментальном стенде приведены на рис. 4.

В стендовых условиях прогрев пористой матрицы производился нагревательным элементом с непостоянной температурой поверхности и переменным коэффициентом теплоотдачи $\alpha$, поэтому зада- ча определения эффективного коэффициента температуропроводности не может быть решена с использованием полученных Г.М. Кондратьевым зависимостей для регулярного режима нагрева [32]. Экспериментально зарегистрированная во всех точках установки термопар скорость изменения температуры $\partial T / \partial t$ строго положительна, изменяется монотонно с различной для каждого из вариантов ориентации нагревательного элемента динамикой. Её расчетное значение определялось по показаниям термопар (рис. 4) через конечноразностную схему $d T / d t$, где $d T$ - изменение показаний термопары за отрезок времени $d t=1 \mathrm{c}$.

В точках установки первой термопары $T_{1}$ для обоих вариантов ориентации нагревательного элемента наблюдалось сначала увеличение скорости изменения температуры во времени до некоторого максимума, а затем плавное её снижение. При расположении нагревательного элемента снизу (рис. $3, a$ ) из-за интенсивных конвективных потоков максимальное значение $d T / d t=0,9 \mathrm{~K} /$ с зарегистрировано на 310 секунде эксперимента. При обратной ориентации нагревательного элемента интенсивность свободной конвекции ниже, и максимальное значение $d T / d t=0,7 \mathrm{~K} /$ с зарегистрировано на 128 секунд позднее.

В остальных точках установки термопар $\left(T_{2}\right.$ и $T_{3}$ ) скорость изменения температуры во времени увеличивается строго монотонно для обоих вариантов расположения нагревательного элемента. Максимальные зарегистрированные значения $d T / d t$ при интенсивной свободной конвекции во второй $T_{2}$ и третьей $T_{3}$ от нагревательного элемента точках составляют 0,6 и $0,3 \mathrm{~K} / \mathrm{c}$. При обратной ориентации нагревательного элемента - 0,18 и $0,06 \mathrm{~K} /$ с соответственно.

При математической обработке экспериментов изменение температуры, которая является определяющей величиной для эффективного коэффициента температуропроводности, в пространстве и времени может быть рассчитано через эффективное уравнение теплового баланса:

$$
\frac{\partial T}{\partial t}=\alpha \frac{\partial^{2} T}{\partial X^{2}},
$$

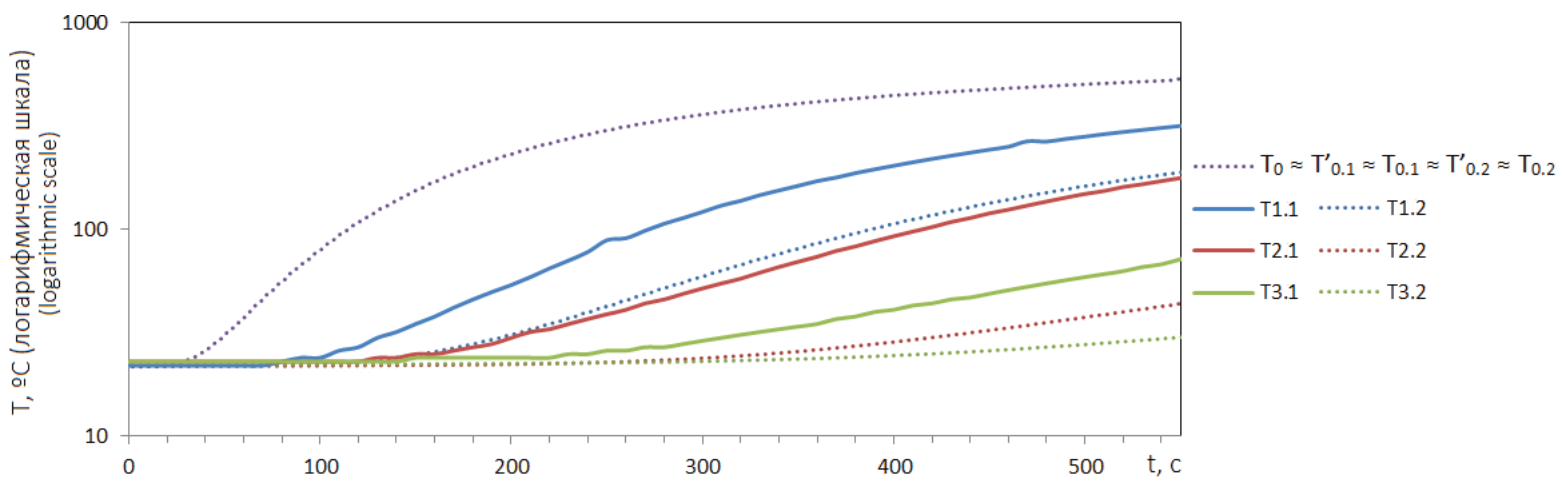

Рис. 4. Показания термопар во время испытаний на экспериментальном стенде

Fig. 4. Thermocouple readings during the experiment 
где $T$ - температура, $\mathrm{K} ; t$ - время, с; $X$ - вертикальная координата в направлении теплопереноса, характеризующая расстояние до нагревательного элемента, отложенное по нормали к его поверхности, м; $\alpha$ - эффективная температуропроводность слоя, $\mathrm{M}^{2} / \mathrm{c}$.

Из (1) следует, что эффективное значение коэффициента температуропроводности прямо пропорционально скорости изменения температуры $\partial T / \partial t$ и обратно пропорционально величине изменения температуры в направлении теплопереноса $\partial^{2} T / \partial X^{2}$, конечноразностный аналог которой:

$$
\frac{\partial^{2} T}{\partial X^{2}}=\frac{T_{1}-2 T_{2}+T_{3}}{h^{2}},
$$

где $T_{1}, T_{2}, T_{3}$ - единовременные показания первой, второй и третьей термопар соответственно, $\mathrm{K} ; h-$ шаг между точками установки термопар по оси $X$, м.

Выразим эффективное значение коэффициента температуропроводности из (1), заменив частные производные конечноразностными схемами:

$$
\alpha=\frac{d T_{2} \cdot h^{2}}{T_{1}-2 T_{2}+T_{3}},
$$

где $d T_{2}$ - изменение показаний второй термопары в единицу времени, $\mathrm{K} / \mathrm{c}(d t=1 \mathrm{c})$.

Подставляя результаты экспериментальных исследований (рис. 4) в (2), получаем зависимости эффективного коэффициента температуропроводности пористого криптола фракции 5-10 мм от температуры (рис. 5). Графики показывают, что истинная температуропроводность криптола сравнительно слабо зависит от температуры и линейно снижается с её ростом. Наряду с этим эффективная температуропроводность пористого криптола с повышением температуры интенсивно растет. Разница в значениях кривых для экспериментов с различной интенсивностью свободной конвекции приводит к выводу, что высокие әффективные значения коэффициентов температуропроводности пористой среды обусловлены в основном конвек- цией. Это хорошо согласуется со взглядами, изложенными А.Ф. Чудновским в [33]. Неподвижные частицы криптола имеют малую площадь соприкосновения друг с другом, поэтому теплоперенос в слое осуществляется главным образом конвективными потоками воздуха. Следовательно, «эффективный коэффициент температуропроводности того же порядка, что и эффективный коэффициент диффузии» [34].

Интегрируя уравнение (1) с подстановкой в него полученных функциональных зависимостей для $a$ (рис. 5), получим значения температуры для всей области интегрирования по координате $\mathrm{x}$ (рис. 6) на момент окончания эксперимента (570 секунда). Значения, определенные на основе выведенных зависимостей, показывают хорошее совпадение температуры в точках замеров на стенде с результатами физического эксперимента для всей области интегрирования по времени.

Следует отметить, что при ориентации нагревательного элемента вниз интенсивная циркуляция воздуха в порах не развивается, т. к. пористая зона лежит ниже его поверхности. Из-за чего при малых значениях температуры теплоперенос обуславливается в основном только теплопроводностью внутри зерен криптола и статичного воздуха в межкусковом пространстве. Эффективное значение температуропроводности пористой засыпки в этом случае на 41 \% ниже её истинного значения, определенного экспериментально. Однако в непосредственной близости к ориентированной вниз поверхности нагревательного элемента (рис. 6) каждые 5-10 мм (размер зерна криптола исследуемой фракции) температура изменяется на 100-200 К. Из-за таких высоких значений температурного градиента в области высоких температур эффективный коэффициент температуропроводности значительно увеличивается по отношению к истинному значению (рис. 5) за счет радиационного теплообмена между твердыми частицами криптола.

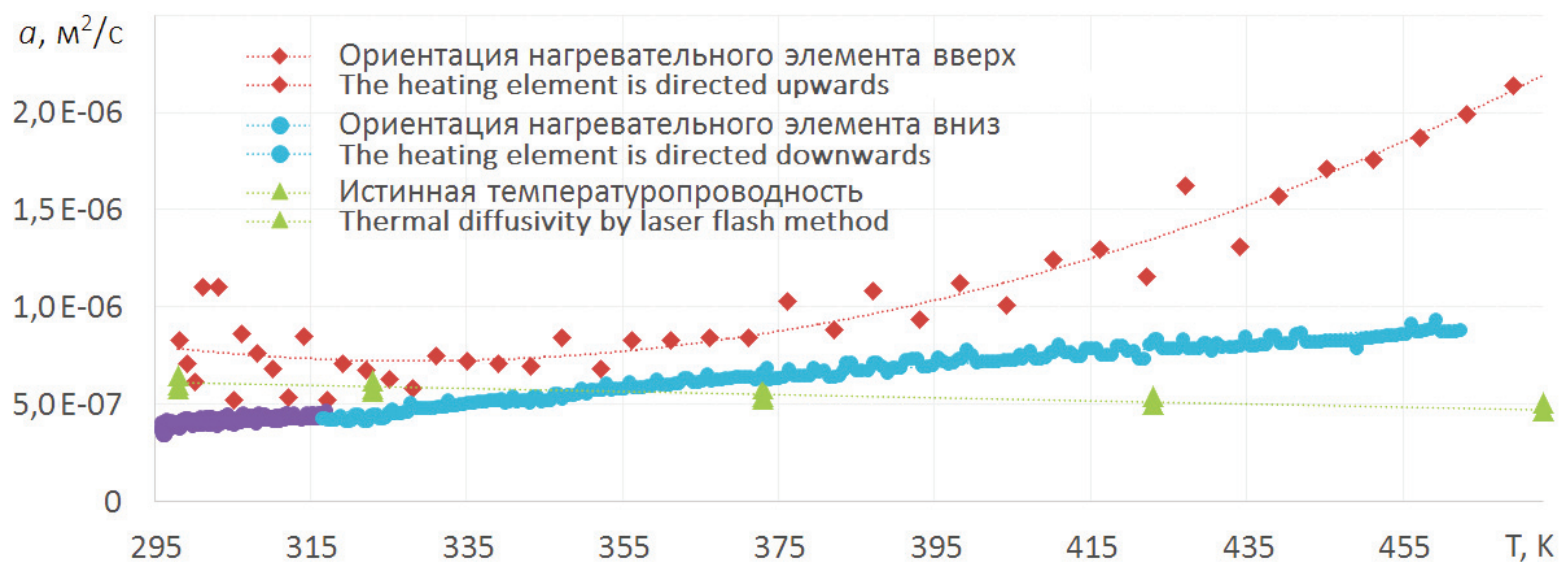

Рис. 5. Аналитическая зависимость коэффициента теплоотдачи $\left(\mathrm{M}^{2} / \mathrm{C}\right)$ от температуры (K)

Fig. 5. Dependence of thermal diffusivity $\left(\mathrm{m}^{2} / \mathrm{s}\right)$ on temperature $(\mathrm{K})$ 


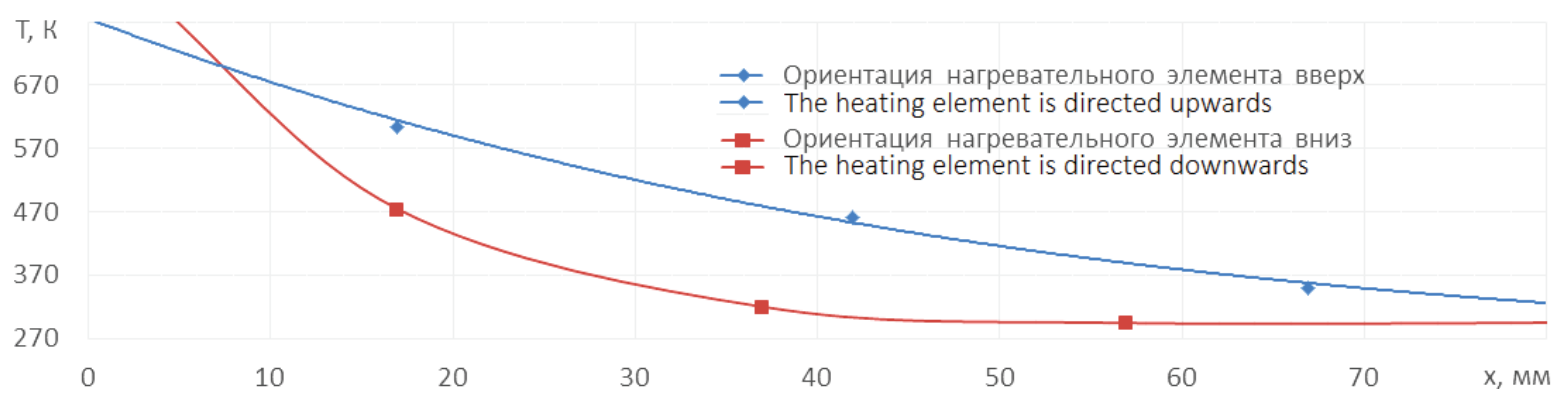

Рис. 6. Изменение температуры (K) по нормали к поверхности нагревательного элемента в направлении теплопереноса на момент времени 570 секунд

Fig. 6. Temperature $(K)$ along the normal to the heating surface in the heat transfer direction at time $t=570 \mathrm{~s}$

\section{Заключение}

В условиях конвективного теплообмена криптола с движущимся вертикально вдоль оси горелки воздухом эффективный коэффициент теплопроводности в горизонтальном направлении значительно ниже, чем в вертикальном, из чего следует важный вывод о том, что горелки исследуемой конструкции могут быть достаточно точно рассчитаны с применением одномерных математических моделей на основе эффективного уравнения теплового баланса (1).

Экспериментально определено истинное значение коэффициента температуропроводности, зависимость которого от температуры с достоверностью $R^{2}=0,924$ описывается линейной функцией:

$$
a=3,15 \cdot 10^{-9}(T-273)+3,14 \cdot 10^{-7} \mathrm{M}^{2} / \mathrm{c} .
$$

Установлено, что в зависимости от расположения и ориентации нагревательного элемента изменяется специфика теплообмена в порах. При ориентации нагревательного элемента вниз из-за низкой интенсивности охлаждения конвективными потоками воздуха его поверхности наблюдаются высокие значения температурного градиента в масштабах зерна криптола исследуемой фракции. Эффективный коэффициент температуропроводности при этом значительно увеличивается по отношению к истинному значению за счет радиационного теплообмена между твердыми частица-

\section{СПИСОК ЛИТЕРАТУРЫ}

1. World Energy Outlook Special Report. Energy and Air Pollution (International Energy Agency). URL: https://www.iea.org/publications/freepublications/publication/ WorldEnergyOutlookSpecialReport2016EnergyandAirPollution.pdf (дата обращения 08.03.2018).

2. Managing the health effects of climate change: Lancet and University College London Institute for Global Health Commission A. Costello, M. Abbas, A. Allen, S. Ball, S. Bell, R. Bellamy, S. Friel, N. Groce, A. Johnson, M. Kett, M. Lee, C. Levy, M. Maslin, D. McCoy, B. McGuire, H. Montgomery, D. Napier, C. Pagel, J. Patel, J.A.P. de Oliveira, N. Redclift, H. Rees, D. Rogger, J. Scott, J. Stephenson, J. Twigg, J. Wolff, C. Patterson // The Lancet. - 2009. - V. 373. - № 9676. - P. 1693-1733.

3. Panwar N.L., Kaushik S.C., Kothari S. Role of renewable energy sources in environmental protection: a review // Renewable and Sustainable Energy Reviews. - 2011. - V. 15. - № 3. P. $1513-1524$ ми криптола. Полученная аналитическая зависимость $\left(R^{2}=0,97\right)$ эффективного значения температуропроводности от температуры для этого случая имеет вид:

$$
a=-8,14 \cdot 10^{-10}(T-273)+6,33 \cdot 10^{-7} \mathrm{M}^{2} / \mathrm{c} .
$$

Для ориентированной вверх горячей поверхности пористая зона лежит выше поверхности нагревательного элемента, и вследствие свободной конвекции в поле сил тяжести во всем объеме криптоловой засыпки развивается активная циркуляция воздуха. Зависимость эффективного значения температуропроводности от температуры с достоверностью $R^{2}=0,924$ в этом случае описывается квадратичной функцией:

$$
a=7 \cdot 10^{-11}(T-273)^{2}-7,74 \cdot 10^{-9}(T-273)+9,37 \cdot 10^{-7} \mathrm{M}^{2} / \mathrm{c} .
$$

Полученные аналитические зависимости эффективного значения температуропроводности от температуры позволят рассчитывать процесс предварительного прогрева криптоловой матрицы с применением дифференциального уравнения теплопроводности для определения расположения и необходимой мощности нагревательных элементов.

Исследование профинансировано Советом по грантам Президента Российской Федерации. Экспериментальные расчеты проведены в Томском Политехническом университете в ралках Гранта програмлы повышения конкурентоспособности Томского политехнического университета (ВИУ-НОЦ И.Н. Бутакова-296/2018).

4. Trend and characteristics of atmospheric emissions of $\mathrm{Hg}, \mathrm{As}$, and Se from coal combustion in China, 1980-2007 / H.Z. Tian, Y. Wang, Z.G. Xue, K. Cheng, Y.P. Qu, F.H. Chai, J.M. Hao // Atmospheric Chemistry and Physics. - 2010. - V. 10. - № 23. P. 11905-11919.

5. Zhang Y., Tao S. Global atmospheric emission inventory of polycyclic aromatic hydrocarbons (PAHs) for 2004 // Atmospheric Environment. - 2009. - V. 43. - № 4. - P. 812-819.

6. Безруких П.П. Возобновляемая энергетика: сегодня - реальность, завтра - необходимость. - М.: Лесная страна, 2007. $120 \mathrm{c}$.

7. Филатов Д.А., Селявский В.Ю. Потенциал использования углеводородокисляющих микроорганизмов для утилизации отработанных масел, а также жидких органических радиоактивных отходов // Перспективы развития фундаментальных наук: сб. науч. трудов XI Междунар. конф. студ. и молодых ученых. - Томск: ТПУ, 2014. - С. 520-523. 
8. Tripathi A.K., Ojha D.K., Vinu R. Selective production of valuable hydrocarbons from waste motorbike engine oils via catalytic fast pyrolysis using zeolites // Journal of Analytical and Applied Pyrolysis. - 2015. - V. 114. - P. 281-292.

9. Catalytic microwave pyrolysis of waste engine oil using metallic pyrolysis char / S.S. Lam, R.K. Liew, C.K. Cheng, H.A. Chase // Applied Catalysis B: Environmental. - 2015. - V. 176-177. № 1. - P. 601-617.

10. BP Statistical Review of World Energy (2015). https://www. bp.com/content/dam/bp-country/es_es/spain/documents/ downloads/PDF/bp-statistical-review-of-world-energy-2015full-report.pdf (дата обращения: 08.03.2018)

11. Чайка О.Г., Ковальчук 0.З., Чайка Ю.А. Мониторинг образования отработанных масел в Украине // Вестник Национального университета «Львовская политехника». - 2009. № 644. - C. 221-224.

12. Glushkov D.0., Strizhak P.A., Vershinina K.Y. Minimum temperatures for sustainable ignition of coal water slurry containing petrochemicals // Applied Thermal Engineering. - 2016. V. $96 .-$ P. 534-546.

13. Hu G., Li J., Zeng G. Recent development in the treatment of oily sludge from petroleum industry: a review // Journal of Hazardous Materials. - 2013. - V. 261. - P. 470-490.

14. Al-attab K.A., Ho J.C., Zainal Z.A. Experimental investigation of submerged flame in packed bed porous media burner fueled by low heating value producer gas // Experimental Thermal and Fluid Science. - 2015. - V. 62. - P. 1-8.

15. Experimental investigation into the combustion characteristics of propane hydrates in porous media / X.-R. Chen, X.-S. Li, Z.-Y. Chen, Y. Zhang, K.-F. Yan, Q.-N. Lv // Energies. - 2015.V. 8. - № 2. - P. 1242-1255.

16. Талантов А.В. Основы теории горения. - Казань: КАИ им. А.Н. Туполева, 1975. - 273 с.

17. Адельсон С.В. Процессы и аппараты нефтепереработки и нефтехимии. - М.: ГНТ Издательство нефтяной и горно-топливной литературы, 1963. - 311 с.

18. Генератор синтез-газа и водорода на основе радиационной горелки / В.С. Арутюнов, В.М. Шмелев, И.Н. Лобанов, Г.Г. Политенкова // Теоретические основы химической технологии. 2010. - № 1. - C. 21-30.

19. Беспламенный способ сжигания твердого топлива: пат. 2393382 РФ МПК F23B70/00; заявл. 06.04.2009; опубл. 27.06.2010, Бюл. № 18. - 10 c.

20. Раяк М.Б., Бернер Г.Я., Кинкер М.Г. Совершенствование процесса сжигания топлива. Обзор зарубежных технологий / / Н0вости Теплоснабжения. - 2011. - T. 135. - № 11. URL: http://www.rosteplo.ru/Tech_stat/stat_shablon.php?id $=2550$ (дата обращения 14.10.2018).
21. Новый тип малоэмиссионных камер сгорания для газотурбинных установок на основе объемных проницаемых матриц / А.Н. Рахметов, В.М. Шмелев, А.А. Захаров, В.С. Арутюнов // Горение и взрыв. - 2013. - Т. 6. - № 6. - С. 61-64.

22. Experimental and numerical study of flameless combustion in a model gas turbine combustor / C. Duwig, D. Stankovic, L. Fuchs, G. Li, E. Gutmark // Combustion Science and Technology. 2008. - V. 180. - № 2. - P. 279-295.

23. Effect of diluted and preheated oxidizer on the emission of methane flameless combustion / S.E. Hosseini, S. Salehirad, M.A. Wahid, M.M. Sies, A. Saat // AIP Conference Proceedings. 2012. - V. 1440. - P. 1309-1312.

24. Astanovsky D.L., Astanovsky L.Z., Kustov P.V. Catalytic oxidation of natural gas using flameless burners of a new design // Catalysis in Industry. - 2013. - V. 5. - № 2. - P. 148-155.

25. Каталог продукции компании Viessmann. URL: http://www.viessmann.com/vires/product_documents / 5681611VSA00001 1.PDF (дата обращения 14.10.2018).

26. Камера сгорания теплогенератора: пат. 2616962 РФ МПК F24C5/00; F23C3/00; F23G7/05; заявл. 27.04.2016; опубл. 18.04.2017, Бюл. № 11. -9 с.

27. Долгов С.В., Хаустов С.А., Табакаев Р.Б. Тестирование конструктивного исполнения технических решений по огневой утилизации жидких углеводородных отходов // Известия Томского политехнического университета. Инжиниринг георесурсов. -2016 . - Т. 327. - № 9. - С. 49-56.

28. Долгов С.В., Клочко К.И., Табакаев Р.Б. Испытания горелочного устройства // Вестник Казанского государственного технического университета им. А.Н. Туполева. - 2014. - № 2. - С. 38-41.

29. Fundamentals of Heat and Mass Transfer / T.L. Bergman, F.P. Incropera, D.P. DeWitt, A.S. Lavine. - USA: John Wiley \& Sons, 2011. - $1048 \mathrm{p}$.

30. Аэродинамические испытания горелочного устройства / А.С. Заворин, А.Ю. Долгих, Р.Н. Фисенко, С.В. Долгов // Известия Томского политехнического университета. - 2013. T. 323. - № 4. - C. 33-37.

31. Волков 0.М. Пожарная безопасность на предприятиях транспорта и хранения нефти и нефтепродуктов. - М.: Недра, 1981. -256 c.

32. Кондратьев Г.М. Регулярный тепловой режим. - М.: Государственное издательство технико-теоретической литературы, 1954. $-408 \mathrm{c}$.

33. Чудновский. А.Ф. Теплообмен в дисперсных средах. - М.: Гостехиздат, 1954. - $444 \mathrm{c}$.

34. Иоффе И.И., Письмен Л.М. Инженерная химия гетерогенного катализа. - Л.: Химия, 1972. - 464 с.

Поступила 30.03.2018 г.

\section{Информация об авторах}

Хаустов C.A., кандидат технических наук, доцент Научно-образовательного центра И.Н. Бутакова Инженерной школы энергетики Национального исследовательского Томского политехнического университета.

Вислогузов P.A., магистрант Научно-образовательного центра И.Н. Бутакова Инженерной школы энергетики Национального исследовательского Томского политехнического университета.

Ивашутенко A.C., кандидат технических наук, доцент отделения электроэнергетики и электротехники Инженерной школы энергетики Национального исследовательского Томского политехнического университета.

Долгов C.B., инженер 1 категории по испытаниям и наладке Муниципального унитарного предприятия г. Нижневартовска «Теплоснабжение».

Tабакаев P.Б., кандидат технических наук, научный сотрудник Научно-образовательного центра И.Н. Бутакова Инженерной школы энергетики Национального исследовательского Томского политехнического университета.

Ермолаев A.H., кандидат технических наук, старший преподаватель кафедры теплогазоснабжения и вентиляции Тюменского индустриального университета. 
UDC 62-69

\title{
EFFICIENT CHARACTERISTICS OF HEAT EXCHANGE IN A POROUS FILLER OF A FLAMELESS HEAT GENERATOR
}

\author{
Sergey A. Khaustov', \\ khaustovSA@tpu.ru
}

Ruslan A. Visloguzov',

visloguzov.ra@yandex.ru

\section{Aleksander S. Ivashutenko', ivashutenko@tpu.ru}

\author{
Sergey V. Dolgov', \\ sergeydolgov555@rambler.ru
}

\section{Roman B. Tabakaev', TabakaevRB@tpu.ru}

\author{
Anton N. Ermolaev ${ }^{3}$, \\ ermolaevanton03@gmail.com \\ 1 National Research Tomsk Polytechnic University, \\ 30, Lenin Avenue, Tomsk, 634050, Russia. \\ ${ }^{2}$ MUE «Teplosnabzhenie», \\ 21, Mendeleev street, Nizhnevartovsk, 628616, Russia. \\ ${ }^{3}$ Tyumen Industrial University, \\ 38, Volodarsky street, Tyumen, 625000, Russia.
}

One of the most common and dangerous emissions polluting the biosphere is liquid hydrocarbon waste. It can contain more than 200 dangerous compounds. A huge pollution rate led to accumulation of more than 1 billion tons of various liquid man-caused wastes. In this connection, one of the priority tasks facing the world community is the problem of utilizing the liquid hydrocarbon wastes. A promising way of solving this problem is the fire disposal of liquid hydrocarbon wastes in flameless heat generators providing its combustion in a cryptol - the porous inert filler. Development of technical solutions for flameless heat generators is now an urgent task, requiring estimating of cryptol thermal-physical characteristics.

The main aim of the research is to determine the thermal-physical characteristics of the cryptol carbon matrix and to derive the functional dependencies for analytical calculation of its preheating.

The methods: cryptol heat capacity and thermal conductivity were measured on the Discovery Flash DLF-1200 thermal diffusivity analyzer in the temperature range of 298 to $573 \mathrm{~K}$. Thermal-physical processes during the flat surface heating of porous filler were experimentally investigated on a specially designed experimental stand. Two variants of the stand layout - with the minimum and maximum convection intensity values - were considered. In the mathematical processing of experimental data, the effective value of the thermal diffusivity was obtained from the finite-difference analog of heat equation.

As a result, the analytical dependences of thermal diffusivity on temperature were obtained. These dependences enable us to use heat equation for calculating the thermal conditions of cryptol preheating.

\section{Key words:}

Liquid hydrocarbon wastes, fire salvaging, porous filler, carbon beads, heat-generating unit using liquid fuel, effective thermal diffusivity.

The research is funded from the Grant Council of the President of the Russian Federation. The experimental calculations are carried out at Tomsk Polytechnic University within the framework of Tomsk Polytechnic University Competitiveness Enhancement Program grant (VIU-SEC I.N. Butakova-296/2018).

\section{REFERENCES}

1. World Energy Outlook Special Report. Energy and Air Pollution (International Energy Agency). Available at: https://www.iea. org/publications/freepublications/publication/ WorldEnergyOutlookSpecialReport2016EnergyandAirPollution.pdf (accessed 8 March 2018).

2. Costello A., Abbas M., Allen A., Ball S., Bell S., Bellamy R., Friel S., Groce N., Johnson A., Kett M., Lee M., Levy C., Maslin M., McCoy D., McGuire B., Montgomery H., Napier D., Pagel C., Patel J., de Oliveira J.A.P., Redclift N., Rees H., Rogger D.,
Scott J., Stephenson J., Twigg J., Wolff J., Patterson C. Managing the health effects of climate change: Lancet and University College London Institute for Global Health Commission. The Lancet, 2009, vol. 373, no. 9676, pp. 1693-1733.

3. Panwar N.L., Kaushik S.C., Kothari S. Role of renewable energy sources in environmental protection: a review. Renewable and $\mathrm{Su}$ stainable Energy Reviews, 2011, vol. 15, no. 3, pp. 1513-1524.

4. Tian H.Z., Wang Y., Xue Z.G., Cheng K., Qu Y.P., Chai F.H., Hao J.M. Trend and characteristics of atmospheric emissions of $\mathrm{Hg}$, As, and Se from coal combustion in China, 1980-2007. At- 
mospheric Chemistry and Physics, 2010, vol. 10, no. 23, pp. 11905-11919.

5. Zhang Y., Tao S. Global atmospheric emission inventory of polycyclic aromatic hydrocarbons (PAHs) for 2004. Atmospheric Environment, 2009, vol. 43, no. 4, pp. 812-819.

6. Bezrukih P.P. Vozobnovlyaemaya energetika: segodnya - realnost, zavtra - neobkhodimost [Renewable energy: today - reality, tomorrow - necessity]. Moscow, Lesnaya strana Publ., 2007. 120 p.

7. Filatov D.A., Selyavsky V.Yu. Potentsial ispolzovaniya uglevodorodokislyayushchikh mikroorganizmov dlya utilizatsii otrabotannykh masel, a takzhe zhidkikh organicheskikh radioaktivnykh otkhodov [Potential for using hydrocarbon oxidizing microorganisms for utilization of used oils, as well as liquid organic radioactive waste]. Sbornik nauchykh trudov XI Mezhdunarodnoy konferentsii studentov i molodykh uchenykh. Perspektivy razvitiya fundamentalnykh nauk [Prospects for development of basic sciences. Proc. of the XI International Conference of students and young scientists]. Tomsk, TPU Publ. house, 2014. pp. 520-523.

8. Tripathi A.K., Ojha D.K., Vinu R. Selective production of valuable hydrocarbons from waste motorbike engine oils via catalytic fast pyrolysis using zeolites. Journal of Analytical and Applied Pyrolysis, 2015, vol. 114, pp. 281-292.

9. Lam S.S., Liew R.K., Cheng C.K., Chase H.A. Catalytic microwave pyrolysis of waste engine oil using metallic pyrolysis char. Applied $\mathrm{Ca}$ talysis B: Environmental, 2015, vol. 176-177, no. 1, pp. 601-617.

10. BP Statistical Review of World Energy (2015). Available at: https://www.bp.com/content/dam/bp-country/es_es/spain/documents/downloads/PDF/bp-statistical-review-of-world-energy2015-full-report.pdf (accessed 8 March 2018).

11. Chayka 0.G., Kovalchuk 0.Z., Chayka Yu.A. Monitoring of formation of used oils in Ukraine. Vestnik Natsionalnogo universiteta «Lvovskaja politehnika», 2009, no. 644, pp. 221-224. In Rus.

12. Glushkov D.0., Strizhak P.A., Vershinina K.Y. Minimum temperatures for sustainable ignition of coal water slurry containing petrochemicals. Applied Thermal Engineering, 2016, vol. 96, pp. 534-546.

13. Hu G., Li J., Zeng G. Recent development in the treatment of oily sludge from petroleum industry: a review. Journal of Hazardous Materials, 2013, vol. 261, pp. 470-490.

14. Al-attab K.A., Ho J.C., Zainal Z.A. Experimental investigation of submerged flame in packed bed porous media burner fueled by low heating value producer gas. Experimental Thermal and Fluid Science, 2015, vol. 62, pp. 1-8.

15. Chen X.-R., Li X.-S., Chen Z.-Y., Zhang Y., Yan K.-F., Lv Q.-N. Experimental investigation into the combustion characteristics of propane hydrates in porous media. Energies, 2015, vol. 8, no. 2, pp. 1242-1255.

16. Talantov A.V. Osnovy teorii goreniya [Fundamentals of the combustion theory]. Kazan, A.N. Tupolev KAI Publ., 1975. 273 p.

17. Adelson S.V. Protsessy $i$ apparaty neftepererabotki i neftekhimii [Processes and apparatuses of oil refining and petrochemistry]. Moscow, Oil and Mining Fuel Literature Publ. house, 1963. 311 p.

18. Arutyunov V.S., Shmelev V.M., Lobanov I.N., Politenkova G.G. A Generator of synthesis gas and hydrogen based on a radiation burner. Theoretical Foundations of Chemical Engineering, 2010, vol. 44 , no. 1, pp. 20-29.
19. Mazurin I.M., Ponomarenko B.A., Mazurin V.K. Besplamenny sposob szhiganiya tverdogo topliva [Flameless method of burning solid fuels]. Patent RF 2393382, 2010.

20. Rayak M.B., Berner G.Ya., Kinker M.G. Improving the combustion process. Overview of foreign technologies. Novosti Teplosnabzheniya, 2011, vol. 135, no. 11. In Rus. Available at: http://www.rosteplo.ru/Tech_stat/stat_shablon.php?id=2550 (accessed 14 October 2018).

21. Rakhmetov A.N., Shmelev V.M., Zakharov A.A., Arutyunov V.S. New type of low-emission combustion chambers for gas turbines based on permeable volumetric matrices. Combustion and explosion, 2013, vol. 6, no. 6, pp. 61-64. In Rus.

22. Duwig C., Stankovic D., Fuchs L., Li G., Gutmark E. Experimental and numerical study of flameless combustion in a model gas turbine combustor. Combustion Science and Technology, 2008, vol. 180, no. 2, pp. 279-295.

23. Hosseini S.E., Salehirad S., Wahid M.A., Sies M.M., Saat A. Effect of diluted and preheated oxidizer on the emission of methane flameless combustion. AIP Conference Proceedings, 2012, vol. 1440 , pp. 1309-1312.

24. Astanovsky D.L., Astanovsky L.Z., Kustov P.V. Catalytic oxidation of natural gas using flameless burners of a new design. Catalysis in Industry, 2013, vol. 5, no. 2, pp. 148-155.

25. Catalog of Viessmann. Available at: http://www.viessmann. com/vires/product_documents/5681611VSA00001_1.PDF (accessed 14 October 2018 ).

26. Dolgov S.V., Savchenko E.K., Tabakaev R.B. Kamera sgoraniya teplogeneratora [Heat generator combustion chamber]. Patent RF 2616962, 2017.

27. Dolgov S.V., Khaustov S.A., Tabakaev R.B. Testing the design of technical solutions for liquid hydrocarbon wastes fire salvaging. Bulletin of the Tomsk Polytechnic University. Geo Assets Engineering, 2016, vol. 327, no. 9, pp. 49-56. In Rus.

28. Dolgov S.V., Klochko K.I., Tabakaev R.B. Tests of the burning device. Vestnik KGTU im. A.N. Tupoleva, 2014, no. 2, pp. 38-41. In Rus.

29. Bergman T.L., Incropera F.P., DeWitt D.P., Lavine A.S. Fundamentals of Heat and Mass Transfer. USA, John Wiley \& Sons, 2011. $1048 \mathrm{p}$.

30. Dolgov S.V., Zavorin A.S., Dolgikh A.Yu., Fisenko R.N. Aerodynamic tests of a burner. Bulletin of the Tomsk Polytechnic University, 2013, vol. 323, no. 4, pp. 33-37. In Rus.

31. Volkov 0.M. Pozharnaya bezopasnost na predpriyatiyakh transporta $i$ khraneniya nefti i nefteproduktov [Fire safety at the enterprises of transport and storage of oil and oil products]. Moscow, Nedra Publ., 1981. 256 p.

32. Kondratev G.M. Regulyarny teplovoy rezhim [Regular thermal conditions]. Moscow, State Publishing House of Technical and Theoretical Literature, $1954.408 \mathrm{p}$.

33. Chudnovsky A.F. Teploobmen v dispersnykh sredakh [Heat transfer in disperse media]. Moscow, Gostekhizdat Publ., 1954. 444 p.

34. Ioffe I.I., Pismen L.M. Inzhenernaya khimiya geterogennogo kataliza [Engineering chemistry of heterogeneous catalysis]. Leningrad, Khimiya publ., 1972. $464 \mathrm{p}$.

Received: 30 March 2018.

\section{Information about the authors}

Sergey A. Khaustov, Cand. Sc., associate professor, National Research Tomsk Polytechnic University.

Ruslan A. Visloguzov, graduate student, National Research Tomsk Polytechnic University.

Aleksander S. Ivashutenko, Cand. Sc., associate professor, National Research Tomsk Polytechnic University.

Sergey $V$. Dolgov, $1^{\text {st }}$ category engineer, MUE «Teplosnabzhenie».

Roman B. Tabakaev, Cand. Sc., researcher, National Research Tomsk Polytechnic University.

Anton N. Ermolaev, Cand. Sc., senior lecturer, Tyumen Industrial University. 